\title{
Stability evaluation of lutein nanodispersions prepared via solvent displacement method: the effect of emulsifiers with different stabilizing mechanisms
}

\begin{abstract}
The stability of lutein nanodispersions was evaluated during storage and when exposed to different environmental conditions. Lutein nanodispersions were prepared using Tween 80, sodium dodecyl sulfate (SDS), sodium caseinate (SC) and SDS-Tween 80 as the emulsifiers. During eight weeks of storage, all samples showed remarkable physical stability. However, only the SC-stabilized nanodispersion showed excellent chemical stability. Under different environmental conditions, the nanodispersions exhibited a varied degree of stability. All nanodispersions showed constant particle sizes at temperatures between 30 and $60{ }^{\circ} \mathrm{C}$. However, at $\mathrm{pH} 2 \mathrm{i} 8$, only the SC-stabilized nanodispersion was physically unstable. The addition of $\mathrm{NaCl}$ (300ї $400 \mathrm{mM}$ ) only caused flocculation in nanodispersion stabilized by SDS-Tween 80. All nanodispersions were physically stable when subjected to different centrifugation speeds. Only Tween 80-stabilized nanodispersion was stable against the effect of freezeï thaw cycles (no phase separation observed). In this study, there was no particular emulsifier that was effective against all of the environmental conditions tested.
\end{abstract}

Keyword: Storage stability; pH; Ionic strength; Centrifugation speed; Heat treatment; Freezeï thaw cycle 